\title{
Condutas de enfermagem para o cuidado à criança com infecção respiratória: validação de um guia*
}

\author{
Nursing conducts for the care of children with respiratory infection: validation of a guideline
}

Conductas de enfermería en el cuidado al niño con infección respiratoria: validación de una guía

\author{
Flávia Paula Magalhães Monteiroㅁ, Viviane Martins da Silva ${ }^{2}$, Marcos Venícios \\ de Oliveira Lopes ${ }^{3}$, Thelma Leite de Araujo ${ }^{4}$
}

\section{RESUMO}

Objetivo: Validar o conteúdo de um guia de condutas de enfermagem específico para crianças com infecção respiratória aguda que apresentam o diagnóstico de enfermagem "Desobstrução ineficaz de vias aéreas". Métodos: Estudo realizado em um hospital pediátrico de Fortaleza-CE, junto a 16 enfermeiras que realizavam o cuidado às crianças e 5 especialistas previamente selecionados. Para validação do conteúdo foi utilizado o Modelo de Fehring. Resultados: As enfermeiras listaram 48 atividades, que foram revisadas e comparadas com as intervenções de enfermagem NIC: Controle de vias aéreas e Monitorização respiratória. Do total, foram selecionadas 26 atividades que foram apresentadas aos especialistas. Estes consideraram 16 atividades válidas para compor o guia de conduta. Conclusão: O guia de conduta desenvolvido apresenta conteúdo válido para as atividades listadas por enfermeiras assistenciais, que apresentam correspondência às apresentadas na NIC.

Descritores: Infecções respiratórias; Cuidados de enfermagem; Estudos de validação; Criança

\begin{abstract}
Objective: To validate the content of a specific nursing guideline for children with acute respiratory infection that presents the 'Ineffective airway clearance' nursing diagnosis. Methods: The study was performed in a pediatric hospital in the city of Fortaleza, with 16 nurses who cared for the children and 5 selected experts. The Fehring Model was used for validation. Results: The nurses listed 48 activities, which were revised and compared with the following NIC interventions: airway control and respiratory monitoring. 26 activities were selected and showed to experts. 16 activities were considered valid to compose the guideline. Conclusion: The guideline developed presents valid content for the activities listed for assistant nurses, which correspond to NIC interventions.
\end{abstract}

Keywords: Respiratory tract infections; Nursing care; Validation studies; Child

\section{RESUMEN}

Objetivo: Validar el contenido de una guía de conductas de enfermería específica para niños con infección respiratoria aguda que presentan el diagnóstico de enfermería "Desobstrucción ineficaz de vías aéreas". Métodos: Se trata de un estudio realizado en un hospital pediátrico de Fortaleza-CE, con 16 enfermeras que brindaban cuidado a los niños y 5 especialistas previamente seleccionados. Para la validación del contenido fue utilizado el Modelo de Fehring. Resultados: Las enfermeras hicieron una lista de 48 actividades, que fueron revisadas y comparadas con las intervenciones de enfermería NIC: Control de vías aéreas y Monitorización respiratoria. Del total, se seleccionaron 26 actividades las cuales fueron presentadas a los especialistas. Éstos consideraron 16 actividades como válidas para componer la guía de conducta. Conclusión: La guía de conducta desarrollada presenta contenido válido para actividades listadas por enfermeras asistenciales, que presentan correspondencia con las presentadas en la NIC.

Descriptores: Infecciones respiratorias; Cuidados de enfermería; Estudios de validación; Niño

\footnotetext{
* Trabalho extraído de parte da monografia de graduação em Enfermagem apresentada à Universidade Federal do Ceará-CE - Fortaleza (CE), Brasil;

${ }^{1}$ Bolsista de Apoio Técnico CNPq. Integrante do Grupo de Estudos em Diagnósticos, Intervenções e Resultados em Enfermagem-GEDIRE- Universidade Federal do Ceará - UFC - Fortaleza (CE), Brasil.

${ }^{2}$ Professora da Faculdade Católica Rainha do Sertão; Pós-graduanda da Universidade Federal do Ceará - UFC. Fortaleza (CE), Brasil.

${ }^{3}$ Doutor, Professor Adjunto do Departamento de Enfermagem da Universidade Federal de Ceará - UFC. Fortaleza (CE), Brasil.

${ }^{4}$ Doutora, Professora Adjunta do Departamento de Enfermagem da Universidade Federal de Ceará / UFC. Fortaleza (CE), Brasil.
} 


\section{INTRODUÇÃO}

As infecções respiratórias agudas têm despertado uma crescente preocupação, devido a ampla abrangência de eventos distintos que comprometem o trato respiratório, além de constituírem uma das principais causas de morbimortalidade em crianças em todo o mundo, sendo as pneumonias responsáveis por cerca de 4 milhões de óbitos por ano nos países em desenvolvimento ${ }^{(1)}$. Esses dados refletem uma grande pressão sobre o setore da saúde e contribuem para a elevação do número de internações hospitalares nas instituições públicas e privadas, resultando em gastos onerosos. Acompanha-se, também, de conseqüências traumáticas para as crianças que são submetidas à terapêutica medicamentosa, muitas vezes endovenosa, ao longo das hospitalizações.

Esses problemas respiratórios acometem as crianças, especialmente nos primeiros cinco anos de vida, pela suscetibilidade e imaturidade do trato respiratório nessa faixa etária. As doenças respiratórias agudas podem se denominadas de acordo com a ocorrência de um processo inflamatório infeccioso (resfriado comum e pneumonias, por exemplo) ou não-infeccioso (rinite alérgica, por exemplo), sofrendo a influência de patógenos, fatores alérgenos e traumas ${ }^{(2)}$. Podem ser classificadas em altas e baixas, diferenciando-se pelo grau de acometimento do trato respiratório. As doenças respiratórias altas têm, em geral, curso benigno e são autolimitadas. Já as doenças respiratórias baixas tendem, a se estender por períodos maiores de tempo e, se não tratadas, podem colocar em risco a vida das crianças ${ }^{(2)}$.

As infecções respiratórias infantis incluem em sua sintomatologia tosse, febre, dispnéia, inflamação orofaríngea, otalgia, anorexia, coriza nasal, tiragem sub e intercostal e cianose ${ }^{(3)}$. Alguns desses sintomas representam agravos decorrentes do processo inflamatório causado pelo acúmulo de secreções, tanto no trato respiratório inferior como no superior, dependendo da região atingida e do agente etiológico, que contribuem para a obstrução das vias aéreas nas crianças.

Quando essas e outras complicações ocorrem, a criança passa a apresentar dificuldade de eliminar secreções e manter a vias aéreas desobstruídas. Tais características compõem os indicadores clínicos do diagnóstico de enfermagem Desobstrução ineficaz das vias aéreas (4), cujos fatores relacionados podem ser ambientais, fisiológicos ou podem estar diretamente relacionados com a via aérea obstruída. Neste âmbito, um conjunto de sinais e sintomas pode surgir de modo isolado ou agrupado e definir situações de desconforto respiratório representadas por episódios esporádicos ou contínuos de dispnéia, expectoração, ruídos adventícios pulmonares, tosse ineficaz ou ausente. Estas alterações exigem uma monitorização constante dos enfermeiros para reverter essa situação de estresse fisiológico no organismo infantil através de medidas de assistência à saúde que englobam um conjunto de ações de enfermagem, que contemplam um cuidado específico, direcionado às peculiaridades desta faixa etária.

Diante disso, a assistência à criança deve contemplar ações sistematizadas que caracterizam o processo de enfermagem, o qual representa um instrumento metodológico que possibilita identificar como uma clientela responde aos problemas de saúde ou aos processos vitais, e determinar que aspectos dessas respostas exigem uma intervenção do profissional de enfermagem ${ }^{(5)}$. O processo de enfermagem funciona como um todo integrado e inter-relacionado, através de etapas que vão desde a coleta de informações, elaboração de diagnósticos, prescrição, implementação e avaliação de enfermagem. Dentre estas etapas, o diagnóstico é o componente essencial, pois representa o elo entre a primeira e as demais etapas, e dá base para o estabelecimento das intervenções e avaliação dos resultados de enfermagem.

As intervenções de enfermagem descrevem um conjunto de atividades específicas ao serem executados os tratamentos de enfermagem, e são planejadas em resposta a um diagnóstico de enfermagem pré-estabelecido, podendo ser vinculadas à Taxonomia da North American Nursing Diagnosis Association (NANDA). A Nursing Intervention Classification (NIC) é uma classificação internacional das intervenções de enfermagem composta por 30 classes, 7 domínios, aproximadamente 500 intervenções compreendidas em principais, sugeridas e opcionais para cada diagnóstico, classificadas em níveis de importância e resolução do mesmo, e mais de 12.000 atividades $^{(0)}$. Estas atividades são definidas como qualquer ação com base no conhecimento científico, realizadas para melhorar os resultados da criança. A classificação das intervenções de enfermagem pode servir de enfoque na elaboração de guias de conduta em enfermagem, os quais fortalecem a prática do enfermeiro por meio de estratégias baseadas em evidências científicas ${ }^{(7)}$.

Os guias de avaliação e conduta, também denominados sistemas de apoio à decisão de enfermagem, são utilizados para aumentar ou estender a capacidade do profissional na tomada de decisão. Esses tomam como base informações que podem facilitar o processo racional do enfermeiro, tanto no cuidado do paciente, abordando a monitorização do cuidado de forma eficiente, como também apoiando o raciocínio diagnóstico e planejamento da assistência de enfermagem, constituindo excelente estratégia para a difusão do uso de terminologias em enfermagem ${ }^{(8)}$. Para o guia ser implementado na prática clínica, ele deve ser submetido a um processo de validação com especialistas na área. Considera-se que, um método é válido quando está bem fundamentado em seus princípios ou evidências, sendo capaz de resistir às críticas ${ }^{(9)}$. 
O processo de validação de intervenções torna-se imprescindível à prática, pois propõe a uniformização e a padronização das ações em enfermagem. Diante disso, considerou-se necessário construir e validar o conteúdo de um guia de condutas em enfermagem direcionado ao diagnóstico Desobstrução ineficaz de vias aéreas, a partir das atividades descritas pela NIC, sugestões de atividades das enfermeiras assistenciais e avaliação por enfermeiros especialistas com base em um modelo decorrente de $\operatorname{validação~}^{(9)}$.

\section{MÉTODOS}

Trata-se de um estudo do tipo exploratório-descritivo com abordagem quantitativa, dividido em três etapas: Levantamento de atividades de enfermagem indicadas por enfermeiras assistenciais que prestavam o cuidado às crianças direcionadas especificamente ao diagnóstico Desobstrução ineficaz das vias aéreas; Identificação, comparação e seleção de atividades descritas na NIC que apresentavam correspondência àquelas levantadas na etapa anterior; Validação das atividades que apresentavam correspondência nas etapas anteriores por um grupo de especialistas.

A primeira etapa foi desenvolvida com enfermeiras que assinaram o Termo de Consentimento Livre e Esclarecido e que trabalhavam na unidade da internação de um hospital pediátrico da periferia de Fortaleza-CE, nos meses de outubro e novembro de 2006. Para a coleta dos dados foi utilizado um instrumento do tipo formulário, contendo dados relativos à identificação pessoal e profissional das enfermeiras, além das atividades de enfermagem indicadas por elas, responsáveis pelo cuidado às crianças que apresentavam o diagnóstico de enfermagem Desobstrução ineficaz de vias aéreas. Neste instrumento havia tópicos referentes às características definidoras do diagnóstico de enfermagem Desobstrução ineficaz das vias aéreas, assim descrito para facilitar a compreensão das enfermeiras do serviço de saúde, já que no referido local de coleta dos dados não se aplica a sistematização da assistência de enfermagem.

No segundo momento, as atividades prescritas pelas enfermeiras foram submetidas a um comparativo com as atividades preconizadas pela NIC para o diagnóstico de enfermagem em estudo.

Para tanto, foi realizada uma análise geral de todas as intervenções propostas pela NIC para o diagnóstico. Foram identificadas 14 possíveis intervenções de enfermagem para a Desobstrução Ineficaz das vias aéreas que apresentavam atividades com alguma correspondência àquelas descritas pelas enfermeiras. Para a análise de correspondência buscou-se identificar palavras diferentes com significados semelhantes, método utilizado em trabalho anterior ${ }^{(10)}$. No final desta etapa, foram selecionadas duas intervenções constantes da NIC, as quais apresentaram maior número de atividades correspondentes entre aquelas identificadas na primeira etapa do estudo. Estas intervenções foram: Controle de vias aéreas e Monitorização respiratória.

$\mathrm{Na}$ etapa seguinte, todas as atividades constantes das duas intervenções selecionadas da NIC e atividades citadas pelas enfermeiras de campo foram submetidas a um painel composto por cinco especialistas, selecionados através de critérios estabelecidos pelos autores deste estudo. Por meio disto, a população foi constituída por enfermeiras qualificadas profissionalmente como doutoras ou mestras em Enfermagem. Neste estudo, foram consideradas especialistas, as enfermeiras que apresentaram o escore mínimo de três pontos, dentre os seguintes critérios, sendo o último considerado critério essencial (Quadro 1).

Quadro 1 - Critérios para seleção de especialistas

\begin{tabular}{|c|c|}
\hline Critérios & Pontos \\
\hline $\begin{array}{l}\text { 1. Ser graduado ou especialista e ter desenvolvido } \\
\text { monografia sobre assistência de enfermagem à } \\
\text { criança ou sobre terminologias de enfermagem }\end{array}$ & 1 \\
\hline 2. Ser mestre em Enfermagem & 1 \\
\hline 3. Ser doutor em Enfermagem & 2 \\
\hline $\begin{array}{l}\text { 4. Ter desenvolvido dissertação de mestrado } \\
\text { envolvendo a temática de terminologias de } \\
\text { enfermagem }\end{array}$ & 1 \\
\hline $\begin{array}{l}\text { 5. Ter desenvolvido dissertação de mestrado } \\
\text { envolvendo a temática de assistência de } \\
\text { enfermagem à criança }\end{array}$ & 1 \\
\hline $\begin{array}{l}\text { 6. Ter desenvolvido tese de doutorado } \\
\text { envolvendo a temática de terminologias de } \\
\text { enfermagem }\end{array}$ & 2 \\
\hline $\begin{array}{l}\text { 7. Ter desenvolvido tese de doutorado } \\
\text { envolvendo a temática assistência de enfermagem } \\
\text { à criança }\end{array}$ & 2 \\
\hline $\begin{array}{l}\text { 8. Ter trabalho publicado relacionado às } \\
\text { terminologias de enfermagem }\end{array}$ & 1 \\
\hline $\begin{array}{l}\text { 9. Ter trabalho publicado relacionado à assistência } \\
\text { de enfermagem à criança }\end{array}$ & 1 \\
\hline $\begin{array}{l}\text { 10. Participar de grupos / projetos de pesquisa } \\
\text { que envolvam a temática terminologias de } \\
\text { enfermagem }\end{array}$ & 1 \\
\hline $\begin{array}{l}\text { 11. Participar de grupos / projetos de pesquisa } \\
\text { que envolvam a temática assistência de } \\
\text { enfermagem à criança }\end{array}$ & 1 \\
\hline $\begin{array}{l}\text { 12. Ser docente do curso de enfermagem em } \\
\text { disciplinas que envolvam a temática terminologias } \\
\text { de enfermagem }\end{array}$ & 2 \\
\hline $\begin{array}{l}\text { 13. Ser docente do curso de enfermagem em } \\
\text { disciplinas que envolvam a temática avaliação e } \\
\text { assistência de enfermagem à criança }\end{array}$ & 2 \\
\hline $\begin{array}{l}\text { 14. Ter no mínimo um ano de experiência } \\
\text { profissional como enfermeiro na assistência a } \\
\text { crianças acometidas por problemas respiratórios. }\end{array}$ & $\begin{array}{l}\text { Critério } \\
\text { essencial }\end{array}$ \\
\hline
\end{tabular}

Estes critérios foram adaptados de um estudo anterior $^{(10)}$. Nesta etapa, para a coleta dos dados foi utilizado um formulário que continha títulos e definições das intervenções: Controle de vias aéreas e Monitorização respiratória com suas respectivas atividades. Os itens para análise estavam apresentados em três colunas: lista das atividades das intervenções de enfermagem relativas às 
crianças; coluna com os escores de 1 a 5 , os quais indicavam: 1 - Atividade inadequada; 2 - Atividade discretamente adequada; 3 - Atividade pouco adequada; 4 - Atividade adequada e 5 - Atividade muito adequada, que deveriam ser marcados com um $\mathrm{X}$ o escore escolhido de acordo com a análise feita; e uma última coluna destinada às sugestões para cada atividade NIC, caso as enfermeiras especialistas considerassem importante fazê-las. A análise dos dados desta etapa seguiu as recomendações do modelo de validação adotado ${ }^{(9)}$. Foram consideradas adequadas aquelas atividades que apresentaram médias ponderadas acima de 0.80, parcialmente adequadas, com médias entre 0.50 e
0.80 e não-adequadas médias abaixo de 0.50 .

A proposta desse estudo foi encaminhada ao Comitê de Ética em Pesquisa, procurando atender aos aspectos contidos na resolução n. ${ }^{\circ} 196 / 96$ sobre pesquisa envolvendo seres humanos do Conselho Nacional de Saúde/Ministério da Saúde ${ }^{(11)}$, obtendo parecer favorável conforme processo $\mathrm{n}^{\circ} .165 / 06$.

\section{RESULTADOS}

Foram listadas 48 atividades de enfermagem desenvolvidas pelas enfermeiras assistenciais na unidade

Tabela 1 - Distribuição das atividades de enfermagem das Intervenções Controle de vias aéreas e Monitorização respiratória para o diagnóstico de enfermagem Desobstrução ineficaz de vias aéreas, validadas pelos enfermeiros especialistas, segundo o modelo de validação de conteúdo de Fehring.

\begin{tabular}{|c|c|c|c|c|c|c|}
\hline \multirow[b]{2}{*}{ Intervenção / Atividades } & \multicolumn{6}{|c|}{ Escore } \\
\hline & $1(=0)$ & $2(=0,25)$ & $3(=0,5)$ & $4(=0,75)$ & $5(=1)$ & $\begin{array}{c}\text { Média } \\
\text { Ponderada }\end{array}$ \\
\hline \multicolumn{7}{|l|}{ 1. Controle de vias aéreas } \\
\hline Posicionar a criança para maximizar o potencial ventilatório. & - & - & 1 & 2 & 2 & 0,80 \\
\hline Remover secreções, encorajando o tossir. & - & - & - & 2 & 3 & 0,90 \\
\hline Encorajar a respiração lenta e profunda, o virar e o tossir. & 1 & & & 3 & 1 & 0,65 \\
\hline $\begin{array}{l}\text { Usar técnicas lúdicas para encorajar a respiração profunda de } \\
\text { crianças. }\end{array}$ & - & 1 & 1 & 2 & 1 & 0,65 \\
\hline Orientar sobre a maneira de tossir eficientemente. & - & - & 1 & 3 & 1 & 0,75 \\
\hline $\begin{array}{l}\text { Auscultar os sons respiratórios, observando as áreas de } \\
\text { ventilação diminuídas ou ausentes e a presença de ruídos } \\
\text { adventícios. }\end{array}$ & - & - & - & - & 5 & 1,00 \\
\hline Administrar broncodilatadores, conforme adequado. & - & - & - & 3 & 2 & 0,85 \\
\hline Administrar tratamentos com aerossol, conforme adequado. & - & - & - & 2 & 3 & 0,90 \\
\hline Administrar ar umidificado ou $\mathrm{O}_{2}$, conforme adequado. & - & - & - & 3 & 2 & 0,85 \\
\hline $\begin{array}{l}\text { Regular a ingesta de líquidos para otimizar o equilíbrio de } \\
\text { líquidos. }\end{array}$ & - & - & - & 4 & 1 & 080 \\
\hline Posicionar de modo a aliviar a dispnéia. & 1 & - & 1 & 1 & 2 & 0,65 \\
\hline \multicolumn{7}{|l|}{ 2. Monitorização respiratória } \\
\hline $\begin{array}{l}\text { Monitorizar a freqüência, ritmo, profundidade e esforço das } \\
\text { respirações. }\end{array}$ & - & - & - & - & 5 & 1,00 \\
\hline $\begin{array}{l}\text { Anotar movimentos do tórax, observando simetria, uso da } \\
\text { musculatura acessória e retrações musculares supraclaviculares } \\
\text { e intercostais. }\end{array}$ & - & - & - & - & 5 & 1,00 \\
\hline Monitorar ruídos respiratórios, tais como sibilos e roncos. & - & - & - & - & 5 & 1,00 \\
\hline $\begin{array}{l}\text { Monitorar padrões respiratórios: bradipnéia, taquipnéia, } \\
\text { hiperventilação, respiração de Kussmaul, de Cheyene-Stocks, } \\
\text { apnêustica, de Biot, e padrões atáxicos. }\end{array}$ & - & 1 & - & 2 & 2 & 0,75 \\
\hline Palpar a expansibilidade pulmonar. & 1 & & 1 & 2 & 1 & 0,60 \\
\hline $\begin{array}{l}\text { Percutir tórax anterior e posterior, dos ápices para as bases, } \\
\text { bilateralmente. }\end{array}$ & - & - & 3 & 2 & - & 0,60 \\
\hline $\begin{array}{l}\text { Auscultar sons respiratórios, observando áreas de ventilação } \\
\text { diminuída/ausente, e presença de ruídos adventícios. }\end{array}$ & - & - & - & 2 & 3 & 0,90 \\
\hline $\begin{array}{l}\text { Auscultar sons pulmonares após tratamentos e anotar } \\
\text { resultados. }\end{array}$ & - & - & - & 3 & 2 & 0,85 \\
\hline Monitorar aumento da agitação, ansiedade e falta de ar. & - & - & & 2 & 3 & 0,90 \\
\hline Monitorar a capacidade da criança para tossir efetivamente. & - & - & 1 & 4 & - & 0,70 \\
\hline Observar início, características e duração da tosse. & - & - & - & 4 & 1 & 0,80 \\
\hline Monitorar secreções respiratórias da criança. & - & - & - & 3 & 2 & 0,85 \\
\hline $\begin{array}{l}\text { Monitorar dispnéia e eventos que possam aumentá-la ou } \\
\text { piorá-la. }\end{array}$ & - & - & - & 5 & - & 0,75 \\
\hline Monitorar laudos de Rx. & - & - & - & 4 & 1 & 0,80 \\
\hline Posicionar a criança lateralmente para prevenir aspiração. & - & - & - & 1 & 4 & 0,95 \\
\hline
\end{tabular}

1. Atividade inadequada 2. Atividade discretamente adequada 3. Atividade pouco adequada 4. Atividade adequada 5. Atividade muito adequada. 
de internação pediátrica. Dentre os problemas que apresentaram maior número de atividades citadas pelas enfermeiras estão a dispnéia e a cianose. Já o problema tosse ineficaz ou ausente apresentou o menor número de atividades. Ressalta-se que, para listar essas atividades, as enfermeiras assistenciais basearam-se em ações já realizadas na instituição.

Em relação ao comparativo realizado com a NIC, para a maioria das atividades, identificou-se correspondência com aquelas citadas pelas enfermeiras, sendo que a nomenclatura utilizada pela NIC abrangia ações mais gerais que incluíam atividades específicas citadas pelas enfermeiras. Foram observadas algumas ações prescritas pelas enfermeiras que necessitavam de um maior detalhamento e adequação. Por outro lado, algumas atividades descritas na NIC foram consideradas não-correspondentes às atividades prescritas pelas enfermeiras, fato este relacionado à questão de tais ações requererem cuidados especializados, exigindo recursos não disponíveis pelo serviço de saúde onde foi realizada a primeira etapa do estudo e ações direcionadas ao cuidado da clientela em estado crítico de saúde.

As 14 intervenções da NIC utilizadas no comparativo somavam um total de 327 atividades. Destas, 63 atividades foram consideradas correspondentes após a análise do comparativo realizado. As intervenções de enfermagem da NIC Controle de vias aéreas e Monitorização respiratória apresentaram maior número de atividades correspondentes, fato este considerado indicativo para a avaliação das intervenções pelos enfermeiros especialistas quanto à adequação das atividades direcionadas às crianças em questão. Ressalta-se que estas intervenções são classificadas pela NIC como prioritárias para o diagnóstico em questão e incluíam um total de 26 atividades diferentes.

Em relação à avaliação pelas enfermeiras especialistas, foram identificadas 16 atividades adequadas e 10 atividades parcialmente adequadas, consideradas de menor peso. A intervenção Controle de vias aéreas apresentou 7 atividades adequadas e 4 atividades parcialmente adequadas, enquanto a intervenção Monitorização respiratória apresentou 9 atividades adequadas e 6 atividades parcialmente adequadas (Tabela 1).

Portanto, das 26 atividades analisadas pelos especialistas, 16 foram validadas de acordo com os parâmetros do modelo adotado, obtendo escore de maior peso, considerado significativo de acordo com os critérios adotados neste estudo.

\section{DISCUSSÃO}

A intervenção de enfermagem Controle de vias aéreas, quando submetida ao processo de validação de conteúdo por especialistas, apresentou 7 atividades adequadas, consideradas de maior peso e importantes no cuidado de crianças com Desobstrução ineficaz de vias aéreas. Essas atividades foram caracterizadas como cuidados básicos de enfermagem, de acompanhamento e melhora do desconforto respiratório, as quais exigem uma avaliação respiratória completa, dependendo do quadro clínico da criança e da eficácia de outras atividades de enfermagem.

Em um estudo realizado com pacientes no pósoperatório imediato, que apresentaram o diagnóstico de enfermagem Desobstrução ineficaz de vias aéreas, foi estabelecido um conjunto de ações denominadas Padrões mínimos de enfermagem: observar padrão respiratório, saturação de oxigênio, avaliação da freqüência e ritmo respiratório, cianose de extremidades, manutenção da nebulização contínua e realização da ausculta pulmonar (12). Esses Cuidados são considerados mínimos e de avaliação contínua do padrão respiratório da criança, similares àqueles cuidados avaliados como adequados por especialistas neste estudo.

As atividades de enfermagem da intervenção Controle de vias aéreas: Remover secreções, encorajando o tossir e Posicionar a criança para maximizar o potencial ventilatório, consideradas adequadas, caracterizam cuidados relevantes para o favorecimento da manutenção das vias aéreas desobstruídas. Estas atividades apresentam considerável importância na resolução do diagnóstico de enfermagem Desobstrução ineficaz de vias aéreas ${ }^{(13)}$. Vale ressaltar que, na execução dessas atividades, o enfermeiro deve, sempre que necessário, incluir o lúdico no sentido de tornar a criança um participante ativo do cuidado.

Para melhorar a permeabilidade das vias aéreas, a remoção das secreções é importante porque as secreções retidas interferem nas trocas gasosas ${ }^{(14)}$. A enfermeira deve atuar removendo as secreções retidas no trato respiratório do cliente, sob seus cuidados, acometido por algum problema de obstrução aérea, no sentido de tornar mais fácil a passagem de ar e promover a troca gasosa ${ }^{(15)}$.

Por outro lado, o posicionamento adequado da criança melhora a expansibilidade pulmonar. A criança exibirá a função respiratória normal quando for posicionada de modo a permitir uma expansão pulmonar máxima, elevando a cabeceira do leito em 30 graus. Além de fornecer umidade aumentada e oxigênio suplementar, colocando a criança em capacetes (lactentes), ou administrar por cateter nasal ou máscaras (crianças maiores) ${ }^{(16)}$.

Após análise do conteúdo por enfermeiras especialistas, as atividades de enfermagem da intervenção Monitorização respiratória adequadas para as crianças menores de cinco anos de idade que apresentam problemas respiratórios agudos, representam ações contínuas de observações do padrão respiratório da criança. A monitoração significa a constante coleta de 
dados selecionados para avaliar se as condições da criança modificaram para melhor, pior ou permanecem dentro do limite normal, proporcionando informações necessárias para determinar as intervenções ${ }^{(10,17)}$. Já, as atividades de monitorização, permitem ao enfermeiro observar as tendências no nível de oxigênio do cliente, capacitando-o a usar critérios objetivos para adaptar a prescrição de enfermagem para melhorar a saturação de oxigênio ${ }^{(16)}$.

\section{CONCLUSÃO}

A construção e validação de um guia de condutas em enfermagem, sob a ótica das intervenções da NIC, tem a intenção de identificar atividades específicas para as crianças acometidas por problemas respiratórios, para que, assim, haja um referencial metodológico mais seguro e inerente à prática de enfermagem.

O guia poderá subsidiar o trabalho do enfermeiro, dando-lhe suporte às decisões clínicas e terapêuticas, além de constituir uma ferramenta para o cuidado às crianças acometidas por infecção respiratória aguda e que apresentam diagnósticos de enfermagem associados ao sistema respiratório.

Diante dos resultados obtidos, acredita-se que este trabalho poderá trazer algumas contribuições para o planejamento dos cuidados às crianças e jovens acometidos por problemas respiratórios e que apresentam o diagnóstico de enfermagem Desobstrução ineficaz de vias aéreas, como também nortear a assistência de enfermagem à criança, proporcionando-lhe um cuidado personalizado e qualificado.

\section{REFERÊNCIAS}

1. Ferreira OS, Britto MCA. Pneumonia aguda: tema que todos devemos estudar. J Pediatr (Rio de J). 2003; 79(6): 478-9.

2. Secretaria de Saúde do Município. Boletim de Saúde de Fortaleza: Doenças respiratórias agudas. Fortaleza: Prefeitura Municipal de Fortaleza; 2002.

3. Barata RCB, Waldman EA, Moraes JC, Guibu IA, Rosov T, Takimoto $S$. Gastroenterites e infecções respiratórias agudas em crianças menores de 5 anos em área da região Sudeste do Brasil, 1986-1987. I - Infecções respiratórias agudas. Rev Saúde Pública = J Public Health. 1996; 30(6): 553-63.
4. North American Nursing Diagnosis Association. Diagnósticos de enfermagem da NANDA: definições e classificações 2003-2004. Porto Alegre: Artmed; 2005.

5. Garcia TR, Nóbrega MML. Sistematização da assistência de enfermagem: reflexões sobre o processo. In: $52^{\circ}$ Congresso Brasileiro de Enfermagem. Recife; 2000.

6. McCloskey JC, Bulechek GM, organizadoras. Classificação das intervenções de enfermagem (NIC). 3a ed. Porto Alegre : Artmed; 2004.

7. Minatel VF, Simões RO. A trilha para demonstrar uma prática baseada em evidências - guias de conduta de enfermagem. In: Bork AMT, Minatel VF, organizadora. Enfermagem de excelência: da visão à ação. Rio de Janeiro: Guanabara Koogan; 2003. p.50-66.

8. Marques IR, Marin HF. Sistemas de apoio à decisão em enfermagem. Rev Paul Enfermagem. 2002; 21(2): 156-62.

9. Fehring RJ. Methods to validate nursing diagnoses. Heart Lung. 1987; 16 (6 Pt 1): 625-9.

10. Napoleão AA. Estudo da aplicabilidade de intervenções da NIC no atendimento a crianças com o diagnóstico de enfermagem "desobstrução ineficaz de vias aéreas relacionada à presença de via aérea artificial" em um centro de terapia intensiva pediátrico [tese]. Ribeirão Preto: Escola de Enfermagem de Ribeirão Preto de Universidade de São Paulo; 2005.

11. Brasil. Ministério da Saúde. Conselho Nacional de Saúde. Normas de pesquisa envolvendo seres humanos - Res. CNS 196/96. Bioética. 1996; 4(2 Supl): 15-25.

12. Martins I, Gutiérrez MGR. Intervenções de enfermagem para o diagnóstico de enfermagem: desobstrução ineficaz de vias aéreas. Acta Paul Enfermagem. 2005; 18(2): 143-8.

13. 13.Napoleão AA. Ineffective airway clearance: applicability of NIC priority interventions in a Brazilian pediatric intensive care unit. Int J Nurs Terminol Classif. 2006; 17(1):76.

14. Smeltzer SC, Bare BG. Brunner \& Suddarth: tratado de enfermagem médico-cirúrgica. 9a ed. Rio de Janeiro: Guanabara Koogan; 2002. v.1.

15. Martins I. Nursing intervention analysis for the nursing diagnosis ineffective airway clearance. In: North American Nursing Diagnosis Association. NANDA, NIC, NOC 2004: Working Together for Quality Nursing Care: Striving Toward Harmonization. Chicago: NANDA International; 2004. p. 107

16. Whaley LF, Wong DL. Enfermagem pediátrica: elementos essenciais à intervenção efetiva. 5a ed. Rio de Janeiro: Guanabara Koogan; 1999.

17. Carpenito-Moyet LJ. Diagnósticos de enfermagem: aplicação à prática clínica. 10a ed. Porto Alegre: Artmed; 2005. 